Luis Javier Capote Pérez

Universidad de La Laguna (University of La Laguna, Spain) e-mail:1capote@ull.es

ORCID: 0000-0002-5066-5874

DOI: 10.15290/mhi.2020.19.01.05

Ma Aránzazu Calzadilla Medina

Universidad de La Laguna (University of La Laguna, Spain) e-mail: acmedina@ull.edu.es

ORCID: 0000-0002-2262-2519

DOI: $10.15290 / \mathrm{mhi} .2020 .19 .01 .05$

\title{
Protección jurídica de la infancia y la adolescencia en España tras la aprobación de la Convención de las Naciones Unidas de los derechos del Niño de $1989^{1}$
}

\begin{abstract}
Legal protection of childhood and adolescence in Spain after the approval of the 1989 Convention on the Rights of the Child

The present paper is about the regulation appliable to childhood and adolescence in the Spanish Law, with special emphasis on the changes derived from the entry into force of the 1978 Spanish Constitution and its influence in the Civil Law area. The article hast two parts. First one begins with the description of origins of the preoccupation for children and teenagers, at the end of the $18^{\text {th }}$ century and the
\end{abstract}

1 Este trabajo constituye uno de los resultados del Grupo consolidado de Investigación Derecho, persona y familia (DERPEFA) de la Universidad de La Laguna. También constituye uno de los resultados del Proyecto de Investigación: "Hacia una revisión del principio de solidaridad familiar: análisis de su alcance y límites actuales y futuros", financiado por PID2019-104226GB-I00/ AEI/10.13039/501100011033 (Ministerio de Ciencia e Innovación/Agencia Estatal de Investigación). España. 
progressive development of politics focused on their protection during the $19^{\text {th }}$ century. Below, there is a brief exposition of the international dimension of scientific and legal treatment, beginning with the celebration of conferences and congresses dedicated to children and continuing with 1924 and 1959 declarations of the rights of the child. Finally, there is another brief description, focused on the 1989 Convention on the Rights of the Child. The second part is centered on the Spanish Law and its rules for the protection of children and teenagers. Firstly, there is a brief historical description, centered over the Spanish perspective in the progressive and growing interest in childhood. Secondly, there is a legal description of Spanish Law rules on the subject, beginning with 1978 Spanish Constitution and continuing with the enumeration of modifications of Civil Law acts, derived from the return of Spain to democracy and the ratification of 1989 Convention.

Key words: Legal protection, childhood, adolescence, risk, distress, welfare institutions

Ut sementem feceris, ita metes

(Lo que siembres será lo que coseches)

Marco Tulio Cicerón (106 a.C. - 43 a.C.)

\section{Antecedentes y consecuencias de la Convención de los Derechos del Niño de 1989}

\subsection{Orígenes de la preocupación y el tratamiento diferenciado de la infancia y la adolescencia}

Las recientes conmemoraciones de la proclamación -20 de noviembre de 1989- y de la entrada en vigor -2 de septiembre de 1990- de la Convención de los Derechos del Niño constituyen buenas oportunidades para llevar a cabo la necesaria reflexión sobre el tratamiento que se ha dado a la infancia y la adolescencia, con anterioridad y posterioridad al hito que supuso la aprobación de este tratado internacional. La Convención es el resultado de un proceso de cambio en todo lo referente a la consideración de las personas menores y en el que la preocupación por su bienestar y el reconocimiento de su singularidad han ido en aumento. En el presente artículo intentaremos exponer cuál ha sido, a grandes rasgos, la incidencia de la Convención en España y en el ordenamiento jurídico español.

Para empezar, hay que recordar que "infancia" y "adolescencia" constituyen conceptos de naturaleza histórica y cultural, a partir de una base de carácter 
biológico ${ }^{2}$. Éstos hacen referencia a un período tan vulnerable como crucial en el crecimiento humano ${ }^{3}$. Sin embargo, la preocupación por infantes y adolescentes es relativamente reciente. Es a finales del S. XVIII que las personas menores de edad son percibidas de otra forma, pasándose de la indiferencia a la preocupación ${ }^{4}$. En este período se pueden encontrar, tanto en Europa como en los Estados Unidos de América, los primeros bosquejos de lo que, tiempo después, habrá de convertirse en los sistemas de protección y de reconocimiento de los derechos de las personas menores ${ }^{5}$. Este interés por la infancia y la adolescencia se enmarca en un cambio, gradual pero importante, en la atención a los colectivos desfavorecidos. Los avances derivados de la revolución industrial y del auge de las ideas ilustradas llevan a los Estados a revisar los sistemas bajo los cuales se atiende a los colectivos más desfavorecidos ${ }^{6}$. El planteamiento de la importancia de la infancia y el reconocimiento del interés del estudio científico de la conducta infantil, serán las bases sobre las que se produzca la reivindicación de la necesidad de organizar y poner en marcha un sistema de protección más eficaz de las personas menores?

Por su parte, el siglo XIX es testigo de un creciente interés por la protección del colectivo que se acentuará durante el siglo XX, incluyendo junto a esa preocupación protectora los objetivos de promoción y participación. Esta inclinación hacia la infancia tiene naturaleza interdisciplinar y acabará desembocando en un movimiento de carácter reformador y vocación de internacionalidad, para conseguir la generalización de unas políticas dirigidas a la protección del colectivo de menores ${ }^{8}$. La influencia reformadora traerá consigo la aparición de normas jurídicas dirigidas, en un primer momento, a la protección de infantes y adolescentes y al refuerzo de la dimensión internacional en lo referente al abordamiento de los problemas relacionados con estos colectivos.

2 I. Ravetllat Ballesté, Protección a la infancia en la legislación española. Especial incidencia en los malos tratos, "Revista de Derecho UNED" 2007, núm. 2, pp. 77-94, p. 79.

3 C. Alemán Bracho, Políticas públicas y marco de protección jurídica del menor en España, "UNED Revista de Derecho Político", mayo-agosto 2014, no 90, pp. 97-134, p. 100 y ss.

4 A.L. Navarro Soto, Los derechos de la infancia: de la protección inicial (en el tiempo de Concepción Arenal) a la promoción actual, "Acciones e Investigaciones Sociales", diciembre de 2012, pp. 189-227, p. 189 y ss.

5 T. Picontó Nogales, Derechos de la infancia: nuevo contexto, nuevos retos, "Derechos y Libertades", Época II, junio de 2009, no 21, pp. 67-93, p. 60.

6 A. Hernández Martín, M. Martínez Muñoz, Derechos de la infancia y tercer sector, "Panorama Social", primer semestre de 2009, 9, pp. 82-90, p. 82 y ss. Este replanteamiento de la relación entre los Estados y su población supone la progresiva asunción, por parte de los poderes públicos, de políticas orientadas hacia las cuestiones sociales y contribuye a la configuración del estado del bienestar.

7 J.M. Fernández Soria, A. Mayordomo Pérez, Perspectiva histórica de la protección a la infancia en España, "Revista Interuniversitaria" 1984, nº 3, pp. 191-214, p. 191.

8 P. Dávila Balsera, L. M. Naya Garmendia, La evolución de los derechos de la infancia: una visión internacional, "Encounters for Education" 2006, 9, pp. 71-93, p. 72. 
Estas dos tendencias seguirán evolucionando a lo largo del siglo XX, incluyendo junto a la protección otras perspectivas, como la de promoción de la infancia y el fomento de la participación de las personas menores en todo aquello que sea de su incumbencia ${ }^{9}$, e implicándoles además, en aquellas cuestiones que interesan globalmente al conjunto de la humanidad, como los objetivos del desarrollo sostenible ${ }^{10}$.

\subsection{La dimensión internacional en la protección de la infancia y la adolescencia}

Como se ha indicado en el apartado anterior, el creciente interés protector por las personas menores derivó prontamente en la asunción de medidas legales para la obtención de estos objetivos, así como en el tratamiento internacional de los mismos. Así, a finales del siglo XIX, podemos encontrar referencias a reuniones, congresos y entidades que estudian y atienden la problemática de la protección a la infancia. En el año 1883 se celebra en París un congreso internacional, auspiciado por la Sociedad General Protectora de la infancia abandonada y culpable. A este evento le seguirán otros de naturaleza similar y centrados en la temática protectora infantil como los de Lyon (1894), Burdeos (1895) o Ginebra (1896). Por su parte, encuentros como el VII Congreso Internacional de Higiene y Demografía, celebrado en Londres en 1892, dedicarán una sección específica al cuidado sanitario de infantes y escuelas. Esta dinámica también se hace presente al otro lado del océano, pues la Casa Blanca estadounidense convocará tres conferencias dedicadas a la salud y la protección de las personas menores en los años 1909, 1919 y $1930^{11}$. Como resultado de los antecedentes anteriores, podemos encontrar la aprobación de varios tratados internacionales que, al fin y a la postre, desembocarían en la Convención de los Derechos del Niño de 1989.

9 El tratamiento de la infancia y la adolescencia ha corrido paralelo al de otros colectivos que, en el pasado, fueron ajenos al interés de la ciencia y de los poderes públicos. De la mencionada indiferencia se pasa al creciente interés por su protección, pero desde un punto de vista en el que las personas menores constituyen el objeto de la preocupación y no sujetos que, pese a hallarse en pleno desarrollo, tienen capacidades cognitivas y volitivas y cuya opinión debe ser escuchada. Aunque su capacidad de obrar siga una evolución paralela a su desarrollo biológico, infantes y adolescentes son personas en el sentido jurídico-civil del térmiNo. Son sujetos de derecho y los derechos que les han sido reconocidos por los distintos instrumentos legales son, sin ningún género de duda, derechos humanos.

10 M.A. Espinosa Bayal, A. Martínez Gimeno, R. García Pérez, La educación para el desarrollo y los derechos de la infancia: el papel de las agencias internacionales y el impacto en la transformación de los contextos, "Educar" 2020, Vol. 56/2, pp. 297-314, p. 299.

11 J.M. Fernández Soria, A. Mayordomo Pérez, op. cit., p. 192. Por otra parte, se puede encontrar una referencia al papel de las asociaciones internacionales en la organización de estos eventos, los cuales se irán celebrando de forma periódica. P. Dávila Balsera, L. M. Naya Garmendia, La evolución de los derechos de la infancia..., p. 76. 
El primer tratado al que debe hacerse mención es la Declaración de Ginebra sobre los Derechos del Niño de 1924, aprobada por unanimidad en la Sociedad de Naciones. Este texto establece, de forma sucinta, una serie de derechos dentro de la dimensión protectora: alimento, cuidado, ayuda, acogida y socorro, educación, reinserción del niño delincuente y protección de los derechos del niño. Es, pues, reflejo de la consideración que el asunto tenía durante el período precedente, donde se hace hincapié en la misión protectora. Se acogen todos los ámbitos de la vida infantil, desde el cuidado biológico, educativo y psicológico al judicial y educativo, pero sin que el colectivo objeto de la norma pueda expresar su opinión ${ }^{12}$.

El siguiente hito en el proceso que habrá de llevar hasta la Convención de 1989 lo encontramos en la Declaración de los Derechos del Niño de las Naciones Unidas de 1959. Este tratado, aprobado por unanimidad por los Estadosmiembro de la organización, surge en un contexto más pacífico, acogiendo los principios establecidos en la Declaración Universal de los Derechos Humanos de 1948 y manteniendo, respecto de la infancia, los ideales de la Declaración de Ginebra de 1924. Además, incorporará un nuevo concepto que se convertirá en una de las bases del tratamiento de infantes y adolescentes desde el punto de vista jurídico: el principio del interés superior del menor ${ }^{13}$. Con ello, se abre el camino hacia la consideración de las personas menores como sujetos cuya voz debe ser atendida, a la hora de tratar los asuntos que a ellos atañen ${ }^{14}$.

\subsection{Aspectos generales de la Convención de las Naciones Unidas de los derechos del Niño de 1989}

La Convención sobre los Derechos del Niño de 1989 fue minuciosamente elaborada durante la década precedente, con la colaboración de representantes de todas las sociedades, todas las religiones y todas las culturas, a propuesta de Polonia. El gobierno de este país sometió a la Comisión de Derechos Humanos de la Organización de las Naciones Unidas una propuesta en este sentido ${ }^{15}$. El resultado fue un tratado que, frente a sus predecesores, no se presenta como un texto meramente declarativo de principios genéricos, sino un instrumento

12 P. Dávila Balsera, L. M. Naya Garmendia, La evolución de los derechos de la infancia..., p. 78. También, P. Dávila Balsera, L. M. Naya Garmendia, La infancia en Europa: una aproximación a partir de los Derechos del Niño, "Revista Española de Educación Comparada" 2003, 9, pp. 83-133.

13 Así, dice en su segundo párrafo el principio VII de la Declaración que el interés superior del niño debe ser el principio rector de quienes tienen la responsabilidad de su educación y orientación; dicha responsabilidad incumbe en primer término a sus padres.

14 P. Dávila Balsera, L. M. Naya Garmendia, La infancia en Europa..., p. 86.

15 P. Dávila Balsera, L. M. Naya Garmendia, La infancia en Europa..., p. 88 y ss. C. del Molino Alonso, El papel de las organizaciones no gubernamentales en la defensa de los derechos de la infancia, "Revista Española de Educación Comparada” 2003, 9, pp. 135-151, p. 140. 
jurídico vinculante donde infantes y adolescentes son considerados sujetos en desarrollo, dotados de derechos pasivos -los vinculados a su protección- pero, también, de derechos activos como las libertades de conciencia, pensamiento, religión, expresión, información o asociación ${ }^{16}$.

La Convención estipula en sus primeros cuarenta y un artículos los derechos humanos de infantes y adolescentes, esto es, de toda persona menor de dieciocho años. Se establecen los mandatos de respeto y protección de los mismos, así como la exigencia de que sean aplicados según los principios rectores contenidos en el texto. Por su parte, los artículos 42 a 45 establecen la obligación, por parte de los Estados Partes, de difundir los principios y las disposiciones del tratado entre su ciudadanía y de llevar a cabo las medidas adecuadas para su efectiva aplicación ${ }^{17}$.

Los derechos de infantes y adolescentes pueden aglutinarse alrededor de los siguientes ámbitos ${ }^{18}$ :

1. Derechos de provisión. Satisfacción de las necesidades básicas de la infancia: salud, nivel de vida, educación, desarrollo de la personalidad y juego.

2. Derechos de identidad y de participación. Garantía de los derechos de la personalidad: nombre, nacionalidad, privacidad, libertad de expresión, libertad de pensamiento, libertad de conciencia, libertad de confesión, derecho de asociación, libertad de opinión y de participación.

3. Derechos de protección. Protección contra toda forma de explotación y violencia: malos tratos, explotación laboral, tráfico de estupefacientes, explotación sexual, venta o trata, tortura y pena de muerte.

Complementariamente a estas categorías, podemos encontrar otros preceptos tendentes a la consecución de la plena efectividad de los derechos indicados.

1. Colaboración con la familia: orientación de progenitores en el ejercicio de los derechos de sus vástagos y promoción de la reunificación familiar.

2. Atención a las personas menores que se hallan en situaciones de vulnerabilidad: secuestro, privación del medio familiar, exilio, discapacidad física o mental, conflictos armados y pertenencia a minorías.

A su vez, los principios rectores de la Convención son los siguientes ${ }^{19}$ :

16 I. Ravetllat Ballesté, Protección a la infancia en la legislación española..., p. 81. C. Villagrasa, Derechos de la infancia y la adolescencia: hacia un sistema legal, "Anales de la Cátedra Francisco Suárez" 2015, 49, pp. 17-41, p. 18.

17 P. Dávila Balsera, L. M. Naya Garmendia, La infancia en Europa..., p. 90.

18 P. Dávila Balsera, L. M. Naya Garmendia, La infancia en Europa..., p. 90 y ss. A. Hernández Martín, M. Martínez Muñoz, op. cit., p. 84.

19 R. García Pérez, L. López Catalán, A. Martínez Gimeno, Defender los derechos de la infancia en el ámbito local. Una práctica innovadora de formación de líderes a favor de la infancia, "International Journal of Educational Research and Innovation" 2020, 13, pp. 74-95, p. 75. 
1. No discriminación: este principio supone la traslación del artículo 2 de la Declaración de los Derechos Humanos de 1948, añadiéndose además la ampliación hacia los supuestos circunstanciales del nacimiento, los impedimentos físicos o cualquier condición de la persona menor o de sus representantes legales.

2. El interés superior del menor: este principio, que, como se ha visto, fue acogido ya en la Declaración de 1959, se consagra aquí como una de las reglas maestras con mayor influencia en el desarrollo jurídico del tratamiento de la infancia y la adolescencia. En el artículo 3 de la Convención se establece que, en todas las medidas concernientes a los niños, que tomen las instituciones públicas o privadas de bienestar social, los tribunales, las autoridades administrativas o los órganos legislativos, una consideración primordial a que se atenderá será el interés superior del niño.

3. La supervivencia y el desarrollo infantil: este principio significa el reconocimiento de las desigualdades sociales y el fomento de la cooperación internacional para poder asegurar y garantizar a las personas menores su pervivencia en condiciones de dignidad.

4. La participación de las personas menores: este principio implica la garantía a infantes y adolescentes del derecho a expresar su opinión libremente, en todos aquellos asuntos que sean de su incumbencia, en función de su edad y de su madurez, fomentando con ello las actitudes democráticas.

La Convención establece un escenario en el que la infancia y la adolescencia han dejado de ser meros destinatarios de la aplicación de normas, instituciones y medidas protectoras, para convertirse en sujetos activos de su existencia, asumiendo en esta fase de desarrollo la puesta en práctica de aquellas facultades que habrán de ejercitar durante la adultez. El tratado reconoce el carácter progresivo en la adquisición de las capacidades intelectuales y cognitivas necesarias para el desarrollo de la capacidad de obrar -entendida aquí en su dimensión jurídica-civil- y, aunque no ha estado exenta de críticas en ciertos pasajes, resulta innegable su condición de hito a nivel internacional en el tratamiento jurídico de las personas, en el período que va desde su nacimiento hasta la mayoría de edad.

\section{Evolución de la protección jurídica de la infancia y la adolescencia en España}

\subsection{La protección jurídica de la infancia y la adolescencia antes de la Constitución Española de 1978}

España no fue ajena al proceso de cambio en la consideración de las personas menores y a la creciente preocupación por las mismas que, como hemos visto, es ya patente en el siglo XIX. Así, podemos encontrar diversos ejemplos de este cambio de actitud, tanto en el plano doctrinal como en el normativo. En el 
primer caso, resulta necesario hablar de la figura de Concepción Arenal Ponte ${ }^{20}$. Esta jurista dedicará buena parte de su vida a la denuncia de las situaciones de los grupos más desfavorecidos, personificando en España las consecuencias de la variación en la consideración de infantes y adolescentes. La infancia es una de las facetas de lo que la autora considera la cuestión social de su siglo, denunciando las prácticas de maltrato y explotación laboral, al considerarlas mucho peor de lo que, también injustamente, se hacía con las personas adultas ${ }^{21}$. Para ella, el niño corría la misma o peor suerte que el adulto en cuanto a protección social, siendo éste, desde su punto de vista, el peor de los males en la España de la época ${ }^{22}$.

Por su parte, en el plano normativo, podemos encontrar también un reflejo de la creciente preocupación por la infancia, en las primeras leyes que tienen por objeto su protección en diversos ámbitos. Así, la Ley del 24 de julio de $1873^{23}$ prohibirá el acceso a ciertos trabajos a niños y niñas menores de cierta edad ${ }^{24}$ y, cinco años más tarde, la Ley de 26 del julio de $1878^{25}$ castigará a quienes sometan a personas menores a ejercicios peligrosos en espectáculos públicos.

Tras el advenimiento de la II República, toma forma la idea de que debe respetarse la futura personalidad de los infantes y adolescentes, sin que se les imponga prematuramente una idea o concepción de la vida que pueda dificultar la libre formación de su conciencia. Esta consideración, que intentaría implantarse en la educación, supone, en cierto sentido un adelanto respecto de lo que, como hemos visto, se ha convertido en idea dominante en el plano internacional: la consideración de las personas menores como sujetos que pueden

20 Concepción Arenal Ponte fue una jurista, pensadora, poeta y dramaturga española, que nació y vivió en Galicia durante el S. XIX. Considerada una de las pioneras del feminismo en España, es también reconocida como una precursora del Trabajo Social en este país y dedicó buena parte de su labor a la denuncia de la situación de colectivos muy desfavorecidos. Suya es la frase «odia al delito y compadece al delincuente» con la que exhortaba a un tratamiento más humanitario de la población reclusa.

21 A.L. Navarro Soto, op. cit., p. 192 y ss.

22 J.M. Fernández Soria, A. Mayordomo Pérez, op. cit., p. 195.

23 Ley de 24 de julio de 1873, publicada en la Gaceta de Madrid no 209, el 28 de julio de 1873. https:// www.boe.es/datos/pdfs/BOE/1873/209/A01193-01193.pdf, (13.09.2020).

24 Este texto legal, conocido como Ley Benot en consideración de su propulsor y redactor, el político y científico gaditano Eduardo Benot Rodríguez, es considerado también como el punto de partida del Derecho del Trabajo en España. A este texto le seguirá el de la Ley de 13 de marzo de 1900, que reforzará la protección de las personas menores en el ámbito laboral, estableciendo en su artículo 1 que los menores de ambos sexos que no hayan cumplido diez años, no serán admitidos en ninguna clase de trabajo. Ley de 13 de marzo de 1900, publicada en la Gaceta de Madrid nº 79, el 14 de marzo de 1900. https://www.boe.es/datos/pdfs/BOE/1900/073/A00875-00876.pdf, (13.09.2020). Esta ley tendrá su desarrollo reglamentario unos meses más tarde, a través del Reglamento para la aplicación de la ley de 13 de Marzo de 1900 acerca del trabajo de mujeres y niños, publicada en la Gaceta de Madrid no 320 de 16 de noviembre de 1900 https://www.boe.es/datos/pdfs/BOE/1900/320/A0057400575.pdf, (13.09.2020).

25 Ley de 26 de julio de 1878, publicada en la Gaceta de Madrid no 209, el 28 de julio de 1878. https:// www.boe.es/datos/pdfs/BOE//1878/209/A00250-00250.pdf, (13.09.2020). 
tomar decisiones en los asuntos que son de su interés y no menos destinatarios de la aplicación de reglas protectoras. El fallido golpe de Estado y la posterior guerra civil que desangró el país entre 1936 y 1939 malograría esta premisa. Y la instauración de la dictadura del general Franco Bahamonde marcaría una aproximación a la infancia basada en los mismos principios rectores e ideologías que formarían un régimen que habría de prolongarse por un espacio de treinta y seis años.

\subsection{La infancia y la adolescencia en la Constitución Española de 1978}

Es incuestionable que el principal hito normativo del ordenamiento jurídico español de las últimas décadas lo constituya la aprobación de la Constitución Española de 1978, pues es en ese momento en el que se instaura un auténtico Estado Democrático de Derecho en el país. La Constitución supuso, entre otras muchas cosas, la derogación de numerosas normas dictadas al amparo del régimen anterior y colocó al país en el camino de la democracia, jurídicamente puede afirmarse que la Constitución marca con claridad un antes y un después en todos los ámbitos.

A grandes rasgos, la Constitución Española de 1978 configura a España bajo los parámetros de un Estado Social y Democrático de Derecho que propugna como valores superiores de su ordenamiento jurídico la libertad, la justicia, la igualdad y el pluralismo político (artículo 1). El Estado se constituye, a su vez, como indisoluble, al tiempo que reconoce y garantiza el derecho a la autonomía de las regiones que la integran y la solidaridad entre todas ellas (artículo 2). Esta organización territorial será determinante a la hora de abordar el tratamiento de la materia objeto de este artículo.

En materia de protección jurídica de la infancia y la adolescencia, ocurre exactamente lo mismo que en el resto de las materias: esta carta magna supone un antes y un después. También se recoge la protección de la familia. El artículo 39 apdo. 1 y 2 del texto constitucional dispone: "1. Los poderes públicos aseguran la protección social, económica y jurídica de la familia. 2. Los poderes públicos aseguran, asimismo, la protección integral de los hijos, iguales éstos ante la ley con independencia de su filiación, y de las madres, cualquiera que sea su estado civil. La ley posibilitará la investigación de la paternidad".

Tras su entrada en vigor se llevó a cabo un proceso normativo que reformó muchas normas, siendo otras también directamente derogadas por no ser constitucionales, como por ejemplo, las normas que preveían diferencias en los derechos que ostentaba la filiación en atención a cuál era su origen (matrimonial, no matrimonial o adoptivo), en la medida en la que la Constitución propugna el principio de igualdad de todos los hijos e hijas ante la Ley, de manera que todos van a ser tratados igual con independencia de cuál sea su filiación, conforme establece el segundo inciso del art. 108 del Código Civil: “(...) La filiación 
matrimonial y la no matrimonial, así como la adoptiva, surten los mismos efectos conforme a las disposiciones de este Código". En cualquier caso, la carta magna recoge la igualdad de todos los españoles en su art. 14: "Los españoles son iguales ante la ley, sin que pueda prevalecer discriminación alguna por razón de nacimiento, raza, sexo, religión, opinión o cualquier otra condición o circunstancia personal o social".

Como norma suprema del ordenamiento jurídico español, la Constitución de 1978 recoge derechos inherentes a la condición de ser humano que, lógicamente también se prevén para las personas menores de edad. Establece obligaciones para los poderes públicos de asegurar la protección social, económica y jurídica de la familia y de los menores de edad, atendiendo a sus particulares circunstancias -como podría ser, por ejemplo, los casos de personas menores de edad con discapacidad o dependientes-.

Por otro lado, impone a los progenitores el deber de asistencia de todo orden a sus hijos e hijas menores de edad, así como en todos los demás casos que en derecho proceda, conforme prevé, con carácter general, el art. 39 apdo. 3 de la carta magna: "Los padres deben prestar asistencia de todo orden a los hijos habidos dentro o fuera del matrimonio, durante su minoría de edad y en los demás casos en que legalmente proceda". El art. 154 del Código Civil dispone específicamente cómo debe articularse dicha asistencia. Concretamente recoge: "Los hijos no emancipados están bajo la patria potestad de los progenitores. La patria potestad, como responsabilidad parental, se ejercerá siempre en interés de los hijos, de acuerdo con su personalidad, y con respeto a sus derechos, su integridad física y mental. Esta función comprende los siguientes deberes y facultades: $10^{\circ}$ Velar por ellos, tenerlos en su compañía, alimentarlos, educarlos y procurarles una formación integral. 2..$^{\circ}$ Representarlos y administrar sus bienes. Si los hijos tuvieren suficiente madurez deberán ser oídos siempre antes de adoptar decisiones que les afecten. Los progenitores podrán, en el ejercicio de su función, recabar el auxilio de la autoridad". Los progenitores representan a sus hijos e hijas menores de edad ${ }^{26}$.

Tanto a nivel internacional como europeo se han ido promulgando normas en las últimas décadas que han recogido distintas cuestiones relacionadas con la protección jurídica de las personas menores de edad, aún de manera indirecta,

26 Art. 162 del Código Civil: "Los padres que ostenten la patria potestad tienen la representación legal de sus hijos menores no emancipados. Se exceptúan: $1 .^{\circ}$ Los actos relativos a los derechos de la personalidad que el hijo, de acuerdo con su madurez, pueda ejercitar por sí mismo. No obstante, los responsables parentales intervendrán en estos casos en virtud de sus deberes de cuidado y asistencia. $2 .^{\circ}$ Aquellos en que exista conflicto de intereses entre los padres y el hijo. $3 .^{\circ}$ Los relativos a bienes que estén excluidos de la administración de los padres. Para celebrar contratos que obliguen al hijo a realizar prestaciones personales se requiere el previo consentimiento de éste si tuviere suficiente juicio, sin perjuicio de lo establecido en el artículo 158”. 
recogiendo distintos derechos. No hay que olvidar que expresamente el art. 39 apdo. 4 del texto constitucional dispone: "Los niños gozarán de la protección prevista en los acuerdos internacionales que velan por sus derechos", además de que, con carácter general prevé en el art. 10.2 de la Constitución Española que: “(...) 2. Las normas relativas a los derechos fundamentales y a las libertades que la Constitución reconoce se interpretarán de conformidad con la Declaración Universal de Derechos Humanos y los tratados y acuerdos internacionales sobre las mismas materias ratificados por España".

La que mayor trascendencia ha tenido, sin duda, es la Convención de las Naciones Unidas de los Derechos del Niño, celebra da en Nueva York el 20 de noviembre de $1989^{27}$, pues los principios que recoge han constituido, en la mayoría de las ocasiones, el punto de partida para la elaboración de normas europeas, estatales y autonómicas. La Convención se ha ratificado prácticamente por todos los países de la Tierra: España lo hizo el 30 de noviembre de 1990. A nivel europeo ha de mencionarse la Carta Europea de los Derechos del Niño de 1992, la Carta de los Derechos Fundamentales de la Unión Europea del 2000, el Tratado de Lisboa de 2009 así como las Orientaciones de la UE relativas a la promoción y la protección de los derechos del menor de 2007.

\subsection{La Ley Orgánica 1/1996, del 15 de enero, de Protección Jurídica del Menor y de modificación parcial del Código Civil y de la Ley de Enjuiciamiento Civil}

Partiendo del marco constitucional descrito, se promulga la Ley Orgánica 1/1996, del 15 de enero, de Protección Jurídica del Menor y de modificación parcial del Código Civil y de la Ley de Enjuiciamiento Civil (en adelante, LO 1/1996), que hoy en día es la norma especial más importante en lo que respecta a la protección jurídica de la infancia y la adolescencia en nuestro país. Resulta muy ilustrativo lo que establece su Exposición de Motivos con relación a los cambios experimentados en la sociedad española pues todos ellos "(...) han provocado un cambio en el estatus social del niño y como consecuencia de ello se ha dado un enfoque a la construcción del edificio de los derechos humanos de la infancia. Este enfoque reformula la estructura del derecho de protección de la infancia vigente en España y en la mayoría de los países desarrollados desde finales del siglo XX y consiste fundamentalmente en el reconocimiento pleno de la titularidad de derechos en los menores de edad y de una capacidad progresiva para ejercerlos".

27 La Convención tiene tres protocolos que la complementan: el protocolo relativo a la participación de los niños en conflictos armados; el protocolo relativo a la venta de niños y la prostitución infantil; y el protocolo relativo a un procedimiento de comunicaciones para presentar denuncias ante el Comité de los Derechos del Niño. 
Dicha norma ha sido reformada desde su aprobación en varias ocasiones, pero, sin duda, la reforma más trascendental que ha experimentado es la operada por la Ley Orgánica 8/2015, del 22 de julio, de modificación del sistema de protección a la infancia y a la adolescencia y por la Ley 26/2015, del 28 de julio, de modificación del sistema de protección a la infancia y a la adolescencia.

En la LO 1/1996, se recoge el principio de protección del interés superior de la persona menor de edad. Este principio, internacionalmente reconocido, se encuentra en la actualidad extensamente regulado en el art. 2 LO 1/1996, reformado en el año 2015, y que, conforme establece la Exposición de Motivos de la LO 8/2015, del 22 de julio, de modificación del sistema de protección a la infancia y a la adolescencia, incorpora “(...) tanto la jurisprudencia del Tribunal Supremo de los últimos años como los criterios de la Observación general n. ${ }^{\circ} 14$, del 29 de mayo de 2013, del Comité de las Naciones Unidas de los Derechos del Niño, sobre el derecho del niño a que su interés superior sea una consideración primordial. Este concepto se define desde un contenido triple. Por una parte, es un derecho sustantivo en el sentido de que el menor tiene derecho a que, cuando se adopte una medida que le concierna, sus mejores intereses hayan sido evaluados y, en el caso de que haya otros intereses en presencia, se hayan ponderado a la hora de llegar a una solución. Por otra parte, es un principio general de carácter interpretativo, de manera que si una disposición jurídica puede ser interpretada en más de una forma se debe optar por la interpretación que mejor responda a los intereses del menor. Pero, además, en último lugar, este principio es una norma de procedimiento. En estas tres dimensiones, el interés superior del menor tiene una misma finalidad: asegurar el respeto completo y efectivo de todos los derechos del menor, así como su desarrollo integral".

De esta manera, será en cada caso concreto en el que se proceda a delimitar el interés de cada una de las personas menores de edad involucradas en el caso, siendo ahora realmente interesante el acudir al citado precepto antes de llevar a cabo su concreción en la práctica pues recoge distintos criterios que van a orientar a los operadores jurídicos en esta difícil tarea, tales como el tener en cuenta, por encima de todo la obligación de proteger su vida, supervivencia y desarrollo, así como la satisfacción de sus necesidades básicas; tener en cuenta sus deseos, sentimientos y opiniones, así como su derecho a participar, en función de su edad, madurez, desarrollo y evolución personal, en el proceso de determinación de su interés superior; el que su vida y desarrollo tenga lugar en un entorno familiar adecuado y libre de violencia, siendo siempre prioritario la permanencia en su familia de origen, salvo que ello no sea posible, preservándose también el mantenimiento de sus relaciones familiares, siempre que sea posible y positivo para el menor; etc. 


\section{Principales derechos y obligaciones de las personas menores de edad en España}

En la actualidad, los derechos más significativos que las personas menores de edad ostentan son los que se reconocen con carácter general en la Convención, si bien algunos se han desarrollado y ampliado. En cualquier caso, siempre ha de partirse del respeto al libre y armónico desarrollo de la personalidad de las personas menores de edad y de, en todo caso, la protección de su interés superior. De esta manera, los progenitores deben velar por los menores de edad en el ámbito de la patria potestad, que debe ser ejercitada conforme a lo preceptuado en el art. 156 del Código Civil ${ }^{28}$.

Los principales derechos son los siguientes: derecho ala vida, a la supervivencia y al desarrollo, derecho a la educación, derecho a la no discriminación, derecho al nombre, derecho a la salud, derecho a la libertad ideológica, derecho a la libertad de expresión, derecho a la información, derecho al honor, a la intimidad y a la propia imagen, derecho de participación, asociación y reunión, derecho a ser oído y escuchado - que implica el poder expresar su opinión en todos los asuntos que le afecten y a que ésta sea tomada en serio y respetada en la medida de lo posible -, derecho a permanecer con su familia de origen siempre que ello sea posible, etcétera.

La persona menor de edad puede, para la defensa y garantía de sus derechos, solicitar la protección y tutela de la entidad pública competente, presentar denuncias individuales al Comité de Derechos del Niño de las Naciones Unidas,

28 Art. 156 CC: "La patria potestad se ejercerá conjuntamente por ambos progenitores o por uno solo con el consentimiento expreso o tácito del otro. Serán válidos los actos que realice uno de ellos conforme al uso social y a las circunstancias o en situaciones de urgente necesidad. Dictada una sentencia condenatoria y mientras no se extinga la responsabilidad penal o iniciado un procedimiento penal contra uno de los progenitores por atentar contra la vida, la integridad física, la libertad, la integridad moral o la libertad e indemnidad sexual de los hijos o hijas comunes menores de edad, o por atentar contra el otro progenitor, bastará el consentimiento de éste para la atención y asistencia psicológica de los hijos e hijas menores de edad, debiendo el primero ser informado previamente. Si la asistencia hubiera de prestarse a los hijos e hijas mayores de dieciséis años se precisará en todo caso el consentimiento expreso de éstos. En caso de desacuerdo, cualquiera de los dos podrá acudir al Juez, quien, después de oír a ambos y al hijo si tuviera suficiente madurez y, en todo caso, si fuera mayor de doce años, atribuirá la facultad de decidir al padre o a la madre. Si los desacuerdos fueran reiterados o concurriera cualquier otra causa que entorpezca gravemente el ejercicio de la patria potestad, podrá atribuirla total o parcialmente a uno de los padres o distribuir entre ellos sus funciones. Esta medida tendrá vigencia durante el plazo que se fije, que no podrá nunca exceder de dos años. En los supuestos de los párrafos anteriores, respecto de terceros de buena fe, se presumirá que cada uno de los progenitores actúa en el ejercicio ordinario de la patria potestad con el consentimiento del otro. En defecto o por ausencia, incapacidad o imposibilidad de uno de los padres, la patria potestad será ejercida exclusivamente por el otro. Si los padres viven separados, la patria potestad se ejercerá por aquel con quien el hijo conviva. Sin embargo, el Juez, a solicitud fundada del otro progenitor, podrá, en interés del hijo, atribuir al solicitante la patria potestad para que la ejerza conjuntamente con el otro progenitor o distribuir entre el padre y la madre las funciones inherentes a su ejercicio". 
plantear sus quejas ante el Defensor del Pueblo, solicitar asistencia legal incluyendo, si fuera el caso, el nombramiento de un defensor judicial, para emprender las acciones judiciales y administrativas necesarias encaminadas a la protección y defensa de sus derechos e intereses -.

La persona menor de edad también es titular de deberes, obligaciones y responsabilidades. Por supuesto, el cumplimiento de los mismos tendrá que ser acorde a la edad y madurez que ostente en cada momento - no es lo mismo un bebé de un año que un niño de 14 años, por ejemplo -. En este sentido, con carácter general, el art. 155 del Código Civil dispone que: "Los hijos deben: $1 .^{\circ}$ Obedecer a sus padres mientras permanezcan bajo su potestad, y respetarles siempre. $2 .^{\circ}$ Contribuir equitativamente, según sus posibilidades, al levantamiento de las cargas de la familia mientras convivan con ella".

Estos deberes se circunscriben, tal y como recoge la Ley, a tres ámbitos diferentes: al familiar (participar en la vida familiar respetando a sus progenitores y hermanos así como a otros familiares; participar y corresponsabilizarse en el cuidado del hogar y en la realización de las tareas domésticas de acuerdo con su edad, con su nivel de autonomía personal y capacidad, y con independencia de su sexo), al ámbito social debiendo respetar a las personas con las que se relacionan y el entorno y; en particular, se debe respetar la dignidad, integridad e intimidad de todas las personas con las que se relacionen con independencia de su edad, nacionalidad, origen racial o étnico, religión, sexo, orientación e identidad sexual, discapacidad, características físicas o sociales o pertenencia a determinados grupos sociales, o cualquier otra circunstancia personal o social; respetar las leyes y normas que les sean aplicables y los derechos y libertades fundamentales de las otras personas, así como asumir una actitud responsable y constructiva en la sociedad; conservar y hacer un buen uso de los recursos e instalaciones y equipamientos públicos o privados, mobiliario urbano y cualesquiera otros en los que desarrollen su actividad; respetar y conocer el medio ambiente y los animales, y colaborar en su conservación dentro de un desarrollo sostenible) y al ámbito escolar (respetar las normas de convivencia de los centros educativos, estudiar durante las etapas de enseñanza obligatoria y tener una actitud positiva de aprendizaje durante todo el proceso formativo; respetar a los profesores y otros empleados de los centros escolares, así como al resto de sus compañeros, evitando situaciones de conflicto y acoso escolar en cualquiera de sus formas, incluyendo el cíber-acoso). 


\section{Familia, persona menor de edad y vulnerabilidad: riesgo y desamparo}

\subsection{Protección y asistencia de las personas menores de edad}

El artículo 20 de la Convención de los Derechos del Niño de 1989 dispone en su párrafo primero que: "Los niños, temporal o permanentemente privados de su medio familiar, o cuyo interés exija que no permanezcan en ese medio, tendrán derecho a la protección y asistencia especiales del Estado". Este derecho se traduce en el ordenamiento jurídico español en un sistema de protección a la infancia y adolescencia que se articula sobre los conceptos de riesgo y de desamparo para los casos en los que la familia no pueda desempeñar correctamente las funciones encomendadas, lo que puede deberse a distintas razones, por lo que los progenitores pueden llegar a verse privados de la patria potestad ${ }^{29}$. Se está, por tanto, ante familias que se encuentran en situación de vulnerabilidad - que podría llegar a constituir la antesala del riesgo o, incluso, del desamparo - y que, por tanto, precisan ayuda y colaboración. Por ello, en cuanto se detecte deben activarse los protocolos existentes según sea el caso, en aras a proporcionar el apoyo requerido y solventar la situación además de eliminar las debilidades encontradas ${ }^{30}$.

La intervención familiar debe seguir los principios de integración y reintegración familiar, como disponen tanto diversos documentos aprobados por el Servicio Social Internacional como las Directrices sobre las modalidades alternativas de cuidado de los niños de la Asamblea General de las Naciones Unidas del 24 de febrero de 2010.

Además de todo lo anterior, existen otras medidas que confluyen en conseguir la ansiada protección. La obligación de las entidades públicas de prestar el apoyo necesario para que las personas menores de edad que se encuentren bajo la patria potestad, tutela, guarda o acogimiento de una víctima de violencia de género o doméstica continúen con ella, así como su protección, atención especializada y recuperación, es una de estas medidas. La Ley Orgánica 1/2004, del 28 de diciembre, de Medidas de Protección Integral contra la Violencia de Género y, específicamente, el Pacto de Estado en materia de Violencia de Género, aprobado por el Congreso de los Diputados el 28 de septiembre de 2017, reconociendo cómo afecta a los hijos e hijas el verse inmersos en una situación de violencia

29 Art. 170 del Código Civil: "El padre o la madre podrán ser privados total o parcialmente de su potestad por sentencia fundada en el incumplimiento de los deberes inherentes a la misma o dictada en causa criminal o matrimonial. Los Tribunales podrán, en beneficio e interés del hijo, acordar la recuperación de la patria potestad cuando hubiere cesado la causa que motivó la privación”. Aún en estos casos de privación o suspensión, los progenitores están obligados a velar por los hijos e hijas menores y a prestarles alimentos, tal y como prevé el art. 110 del Código Civil.

$30 \mathrm{Al}$ respecto, no hay que olvidar que la pobreza de los progenitores, de las personas tutoras o guardadoras no podrá ser tenida en cuenta para la valoración de la situación de desamparo. 
de género, propone actuaciones importantes, tales como la suspensión del régimen de visitas cuando los hijos presencien, sufran o convivan con manifestaciones de violencia, prohibiéndose que las personas menores de edad vayan a ver a prisión al padre condenado, así como que no se pueda adoptar, ni siquiera de manera provisional, la custodia compartida para padres maltratadores incluso cuando haya un procedimiento penal y si existe orden de alejamiento. También destaca el Convenio relativo a la Protección de los Niños contra la explotación y abuso sexual del 25 de octubre de 2007 y la Directiva del Parlamento Europeo $y$ del Consejo 2011/93/UE, del 13 de diciembre de 2011, relativa a la lucha contra los abusos sexuales y la explotación sexual de los menores y la pornografía infantil. Además, es importante señalar que a nivel nacional se ha impulsado un proyecto de Ley Orgánica de protección integral a la infancia y la adolescencia frente a la violencia, que se encuentra en la actualidad (septiembre de 2020) tramitándose en las Cortes Generales ${ }^{31}$.

Por último, también ha de destacarse que las entidades públicas tienen la obligación de ofrecer, a jóvenes tutelados próximos a cumplir la mayoría de edad, programas de preparación para la vida independiente (art. 22 bis LO 1/1996). Con esta medida se trata de solucionar un problema que existía antes de su introducción: que los jóvenes alcanzaran la mayoría de edad en el centro en el que vivían sin estar preparados para vivir de forma independiente debiendo, además, abandonar el centro precisamente por ser ya mayores de edad.

\subsection{Situación de riesgo y situación de desamparo}

Conforme a lo previsto en el artículo 17 LO 1/1996, habrá una situación de riesgo cuando por circunstancias, carencias o conflictos familiares, sociales o educativos, la persona menor de edad se vea perjudicada en su desarrollo personal, social, familiar o educativo, en su bienestar o en sus derechos de forma que, sin alcanzar la entidad, intensidad o persistencia que fundamentarían su declaración de situación de desamparo y la asunción de la tutela por ministerio de la ley, sea precisa la intervención de la administración pública competente, para eliminar, reducir o compensar las dificultades o inadaptación que le afectan y evitar su desamparo y exclusión social, sin tener que ser separado de

31 Disponible en: http://www.congreso.es/public_oficiales/L14/CONG/BOCG/A/BOCG-14-A-22-1. PDF (13.09.2020). En la Exposición de Motivos de dicho Proyecto de Ley se recoge: "Esta ley combate la violencia sobre la infancia y la adolescencia desde una aproximación integral, en una respuesta extensa a la naturaleza multidimensional de sus factores de riesgo y consecuencias. La ley va más allá de los marcos administrativos y penetra en numerosos órdenes jurisdiccionales para afirmar su voluntad holística. Desde una perspectiva didáctica, otorga una prioridad esencial a la prevención, la socialización y la educación, tanto entre las personas menores de edad como entre las familias y la propia sociedad civil. La norma establece medidas de protección, detección precoz, asistencia, reintegración de derechos vulnerados y recuperación de la víctima, que encuentran su inspiración en los modelos integrales de atención identificados como buenas prácticas a la hora de evitar la victimización secundaria”. 
su entorno familiar ${ }^{32}$. Ante una posible situación de riesgo, deberá articularse un proyecto de actuación que debe ser consensuado con los progenitores u otros responsables legales del menor de edad, pues de no existir ese consenso, se declarará la situación de riesgo del menor mediante resolución administrativa motivada.

Por su parte, según lo previsto en el artículo 18 LO 1/1996, la situación de desamparo se da ante el incumplimiento o el imposible o inadecuado ejercicio de los deberes de protección establecidos para la guarda de los menores, cuando éstos queden privados de la necesaria asistencia moral o material ${ }^{33}$, de manera que la persona menor de edad declarada en desamparo será tutelada por la entidad pública. Para que se declare el desamparo, se requiere que se dé alguna circunstancia que ostente la suficiente gravedad en la medida en la que, valorada y ponderada conforme a los principios de necesidad y proporcionalidad, suponga una amenaza para la integridad física o mental del menor.

\subsection{Instituciones de protección del menor de edad}

En primer lugar, han de señalarse las instituciones que posibilitan una colaboración de la entidad pública con la familia, tal y como ocurre con la guarda voluntaria; o con parientes, amistades o allegados, como se plantea en la guarda de hecho que, correctamente ejercida, impide el desamparo ${ }^{34}$.

32 Constituye una situación de riesgo la negativa a proporcionar la asistencia sanitaria necesaria para salvaguardar la vida o integridad física o psíquica de un menor. El tener un hermano en dicha situación (salvo que las circunstancias familiares hayan cambiado de forma evidente) se considerará indicador de riesgo, así como la concurrencia de circunstancias o carencias materiales.

33 Conforme a lo previsto legalmente, constituyen situaciones de desamparo: el abandono del menor -bien porque falten las personas a las que por ley corresponde el ejercicio de la guarda, o bien porque éstas no quieran o no puedan ejercerla; el transcurso del plazo de guarda voluntaria, bien cuando sus responsables legales se encuentren en condiciones de hacerse cargo de la guarda del menor y no quieran asumirla, o bien cuando, deseando asumirla, no estén en condiciones para hacerlo, salvo los casos excepcionales en los que la guarda voluntaria pueda ser prorrogada más allá del plazo de dos años-; el riesgo para la vida, salud e integridad física del menor; el incumplimiento o el imposible o inadecuado ejercicio de los deberes de guarda como consecuencia del grave deterioro del entorno o de las condiciones de vida familiares, cuando den lugar a circunstancias o comportamientos que perjudiquen el desarrollo del menor o su salud mental; el riesgo para la salud mental del menor, su integridad moral y el desarrollo de su personalidad debido al maltrato psicológico continuado o a la falta de atención grave y crónica de sus necesidades afectivas o educativas por parte de progenitores, tutores o guardadores; la inducción a la mendicidad, delincuencia o prostitución, o cualquier otra explotación del menor de similar naturaleza o gravedad; la ausencia de escolarización o falta de asistencia reiterada y no justificada adecuadamente al centro educativo y la permisividad continuada o la inducción al absentismo escolar durante las etapas de escolarización obligatoria; cualquier otra situación gravemente perjudicial para el menor que traiga causa del incumplimiento o del imposible o inadecuado ejercicio de la patria potestad, la tutela o la guarda, cuyas consecuencias no puedan ser evitadas mientras permanezca en su entorno de convivencia.

34 De Pablo Contreras, P. V., Situaciones de desamparo y situaciones de riesgo de desprotección social de los menores en la Ley Orgánica 1/1996, del 15 de enero, de Protección Jurídica del Menor, [in:] Desprotección social de los menores y las instituciones de amparo reguladas en la ley orgánica de protección jurídica del menor: Jornadas de derecho civil en homenaje a Estanislao de Aranzadi, coord. M. A. Pérez Álvarez, 1997, pp. 49-62. 
Produciéndose ya situaciones de necesidad del menor de edad no atendidas, surge la obligación de las entidades públicas de llevar a cabo la atención inmediata que precise cualquier menor, que puede articularse en un primer momento y sin que haya previa declaración de desamparo ni solicitud expresa de los progenitores en dicho sentido, bajo su guarda provisional para atender a situaciones de urgencia. De ya encontrarse en situación de desamparo, procede la asunción de la tutela automática, ex lege, sin dilación, para poder proteger así a la persona menor de edad. Quienes estén bajo acogimiento - tanto familiar como residencial - tienen una serie de derechos específicos expresamente recogidos en la Ley (artículo 21 LO 1/1996).

Conforme a lo que dispone el artículo 20 LO 1/1996, el acogimiento familiar produce la plena participación de la persona menor de edad en la vida de la familia que lo acoge e impone a quien lo recibe las obligaciones de velar por él, tenerlo en su compañía, alimentarlo, educarlo y procurarle una formación integral en un entorno afectivo. Podrá tener lugar, una previa valoración de la familia, en la propia familia extensa o en familia ajena. Por su parte, el acogimiento residencial (artículo $21 \mathrm{LO}$ 1/1996) se articula mediante un proyecto socio-educativo individual en pro de la persona menor de edad en el marco del plan individualizado de protección que debe recoger la finalidad del ingreso, los objetivos a conseguir y el plazo para su consecución. Esta medida es subsidiaria a la de acogimiento familiar, especialmente para personas menores de seis años. Si se trata de menores de tres años, no podrá acordarse, salvo en supuestos de imposibilidad, debidamente acreditada, de adoptar en ese momento la medida de acogimiento familiar o cuando esta medida no convenga al interés superior de la persona menor de edad.

Es muy interesante señalar una reciente previsión normativa que articula lo que se ha denominado acogimiento residencial de menores con problemas de conducta que se llevará a cabo en centros específicos. Se trata de una medida a utilizar una vez descartadas las restantes, esto es, siempre como último recurso, en tanto en cuanto, no hay que olvidar que se limitan derechos fundamentales.

Por último, la adopción (artículo 175 y ss. del Código Civil) se erige en la institución jurídica de mayor trascendencia en la medida en la que la misma es irrevocable, dado que con su constitución se crean, entre la persona adoptante y la adoptada, los mismos vínculos jurídicos existentes entre los progenitores (y sus familias de éstos) y sus hijos biológicos, y se extinguen, salvo algunos, todos los vínculos jurídicos entre la persona adoptada y su familia biológica. Es novedosa la introducción mediante la reforma del 2015, en aras a facilitar de estabilidad familiar a algunos menores, sobre todo a los mayores, de lo que se conoce como "adopción abierta", que es la adopción en la que continúa la relación (contacto) con algún o algunos miembros de la familia de procedencia, normalmente a través de visitas regulares o de comunicaciones (email, llamadas telefónicas, etc.). También se prevé la posibilidad de adoptar fuera de España. 
La adopción internacional se encuentra regulada por la Ley 54/2007, del 28 de diciembre, de Adopción Internacional que la define como aquella institución en la que un menor considerado adoptable por la autoridad extranjera competente y con residencia habitual en el extranjero, es o va a ser desplazado a España por adoptantes con residencia habitual en España, bien después de su adopción en el Estado de origen, o bien con la finalidad de constituir tal adopción en España.

\section{Conclusiones}

La proclamación de la Convención de los Derechos del Niño en 1989 y su posterior entrada en vigor al año siguiente, con la ratificación por parte de una veintena de países, entre los cuales se encontraba España, constituyen, junto a la promulgación de la vigente Constitución Española, dos hitos en el tratamiento que el ordenamiento jurídico hispánico da a la infancia y la adolescencia. En ese sentido, resulta una afortunada casualidad que el decenio de preparación del texto del tratado internacional coincidiera con los diez primeros años de regreso a la democracia, bajo la égida de la carta magna del 6 de diciembre de 1978. Así pues, el tratamiento que desde el Derecho español se da al colectivo compuesto por las personas menores se elabora introduciendo unos principios rectores que están presentes en ambos textos legales y que contrastan poderosamente con las ideas-fuerza que regían la normativa aplicable en la materia antes de sus respectivas entradas en vigor.

Como se ha visto, la Constitución Española reconoce la igualdad de los distintos modelos familiares y la igualdad en la descendencia, entendiendo por tal la no discriminación por razón de origen. Estas reglas marcan el punto de partida de una progresiva introducción del Derecho Público en un área tradicionalmente privada, como había sido el Derecho de Familia, la cual constituía, desde cierto punto de vista, el hogar ancestral del tratamiento a la infancia y la adolescencia. La promulgación de las distintas leyes autonómicas en la materia viene, a su vez, a unir a este creciente interés público la consideración de las personas menores como algo más que meros objetos de la normativa protectora, para convertirles, en desarrollo de la LO 1/1996, en sujetos con una capacidad en desarrollo para regir sus destinos, cumpliendo con ello, al menos sobre el papel, con el mandato establecido en la Convención de 1989. No obstante, no debe perderse de vista la necesidad de recordar que las normas jurídicas no son metas y objetivos en sí mismas, sino puntos de partida para alcanzar los resultados previstos o anhelados en sus preámbulos y articulados. Situaciones tan complicadas, como la crisis económica que estalló en $2008^{35}$,

35 Sobre los nefandos efectos de esta crisis en el sistema, vid. G. De Castro Lamela, C. Pasqual Roig, La regresión de los derechos de la infancia en España 2007-2013, "XI Congreso Internacional de la Infancia Maltratada", 2015. 
o tan dramáticas como la pandemia generada por el COVID-19, han puesto a prueba la eficacia práctica del sistema estructurado a partir de la carta magna de 1978 y de la ratificación del tratado de 1989 e invitan a la necesaria reflexión en torno a su efectividad, tanto en condiciones normales como en la extraña y atípica "nueva normalidad" en la que vivimos actualmente.

\section{Bibliography}

Alemán Bracho C., Políticas públicas y marco de protección jurídica del menor de España, "UNED Revista de Derecho Político" 2014, 90.

Berrocal Lanzarot A., Las instituciones de protección a la infancia y adolescencia: atención inmediata, situación de riesgo y desamparo, tutela administrativa, y acogimiento familiar y residencial tras la Ley 26/2015, "LA LEY Derecho de familia" 2014 (13 de octubre).

Botana García G., La Ley 26/2015, de 28 de julio, de modificación del sistema de protección a la infancia y a la adolescencia, "Actualidad Civil" 2014, 9.

Calzadilla Medina M. A., La adopción internacional. "Código Civil Comentado", segunda edición, Vol. I, Cizur Menor 2016.

Calzadilla Medina M. A., Lo que la patria potestad no ampara, "Revista Aranzadi de Derecho de Familia" 2017, 74.

Dávila Balsera P., Naya Garmendia L. M., La infancia en Europa: una aproximación a partir de los Derechos del Niño, "Revista Española de Educación Comparada 2003, 9.

Dávila Balsera P., Naya Garmendia L.M., La evolución de los derechos de la infancia: una visión internacional. "Encounters on Education" 2006, 7.

Defensor del Pueblo, Informe sobre los centros de protección de menores con trastornos de conducta y en situación de dificultad social, Madrid 2009, (accesible en https://www.defensordelpueblo.es/wp-content/uploads/2015/05/2009-01-Centros-de-protecci\%C3\%B3n-de-menores-con-trastornos-de-conducta-y-en-situaci\%C3\%B3n-de-dificultad-social.pdf) (13.09.2020).

De Castro Lamela G., Pasqual Roig C., La regresión de los derechos de la infancia en España 2007-2013, "XI Congreso Internacional de la Infancia Maltratada" 2015.

De la Iglesia Monje M. I., Ley Orgánica de modificación del sistema de protección a la infancia y adolescencia: las garantías de una protección uniforme a los menores más vulnerables en base a su supremo interés, "Diario La Ley" 2015 (24 de julio), núm. 8590.

Del Molino Alonso C., El papel de las organizaciones no gubernamentales en la defensa de los derechos de la infancia, "Revista Española de Educación Comparada" 2003, 9.

De Pablo Contreras P.V., Situaciones de desamparo y situaciones de riesgo de desprotección social de los menores en la Ley Orgánica 1/1996, del 15 de enero, de Protección 
Jurídica del Menor, [in:] Desprotección social de los menores y las instituciones de amparo reguladas en la ley orgánica de protección jurídica del menor: Jornadas de derecho civil en homenaje a Estanislao de Aranzadi, coord. M.A. Pérez Álvarez, 1997.

Espinosa Bayal M.A., Martínez Gimeno A., García Pérez R., La educación para el desarrollo y los derechos de la infancia: el papel de las agencias internacionales y el impacto en la transformación de los contextos, "Educar" 2020, Vol. 56/2.

Fernández Soria J. M., Mayordomo Pérez A., Perspectiva histórica de la protección a la infancia en España, "Revista Interuniversitaria" 1984, 3.

García Pérez R., López Catalán L., Martínez Gimeno A., Defender los derechos de la infancia en el ámbito local. Una práctica innovadora de formación de líderes a favor de la infancia, "International Journal of Educational Research and Innovation” 2020, 13.

Gómez Megías A.M., Aspectos básicos de la Ley Orgánica 8/2015, de 22 de julio, de modificación del sistema de protección de la infancia y adolescencia, "Diario La Ley" 2015 (24 de julio), núm. 8590.

Hernández Martín A., Martínez Muñoz M., Derechos de la infancia y tercer sector, "Panorama Social" 2009 (primer semestre), 9.

López Azcona A., Luces y sombras del nuevo marco jurídico en materia de acogimiento y adopción de menores: a propósito de la Ley Orgánica 8/2015 y la Ley 26/2015 de modificación del sistema de protección a la infancia y adolescencia, "Boletín del Ministerio de Justicia” 2016, Año LXX, 2185.

López M., Del Valle J. F., Montserrat C., Bravo A., Niños que esperan. Estudio sobre casos de larga estancia en acogimiento residencial, Ministerio de Sanidad, Política Social e Igualdad, Madrid 2010.

Martínez de Aguirre y Aldaz, C., La protección jurídico-civil de la persona por razón de la menor edad (Una aproximación teleológica a las instituciones de asistencia y protección de menores en nuestro Derecho civil), "Anuario de Derecho Civil" $1992,4$.

Navarro Soto A.L., Los derechos de la infancia: de la protección inicial (en el tiempo de Concepción Arenal) a la promoción actual, "Acciones e Investigaciones Sociales" 2012, 32.

Paniza Fullana A., La modificación del sistema de protección a la infancia y a la adolescencia: La Ley Orgánica 8/2015, de 22 de julio y la Ley 26/2015, de 28 de julio, "Aranzadi Civil-Mercantil" 2015, Vol. 2, 8.

Parra Lucán M.A., La voluntad y el interés de las personas vulnerables. Modelos para la toma de decisión en asuntos personales, Centro de Estudios Ramón Areces, Madrid 2016.

Pérez Cebadera M. A., El ingreso de menores con problemas de conducta en centros de protección específicos, "Práctica de Tribunales" 2017, 124.

Picontó Nogales T., Derechos de la infancia: nuevo contexto, nuevos recursos, "Derechos y libertades" 2009, 21.

Ravetllat Ballesté I., Protección a la infancia en la legislación española. Especial incidencia en los malos tratos, "Revista de Derecho UNED” 2007, núm. 2. 
Ravetllat Ballesté I., El interés superior del niño a la luz del nuevo artículo 2 de la Ley Orgánica 1/1996, de 15 de enero, "LA LEY Derecho de familia" 2015 (13 de octubre).

Rivero Hernández F., El interés del menor, segunda edición, Dykinson, Madrid 2007.

Rodrigo López, M.J. (coord.), Situación de la infancia y la familia en Canarias, Wolters Kluwer, Madrid 2018.

Villagrasa C., Derechos de la infancia y la adolescencia: hacia un sistema legal, "Anales de la Cátedra Francisco Suárez" 2015, 49.

Yzquierdo Tolsada M., Patria potestad, [en:] Tratado de derecho de la familia, Las relaciones paterno-filiales (II). La protección penal de la familia, dir. M. Yzquierdo Tolsada, M. Cuena Casas, 2011.

\section{SUMmary}

\section{Legal protection of childhood and adolescence in Spain after the approval of the 1989 Convention on the Rights of the Child}

Legal protection of childhood and adolescence in Spain after the approval of the 1989 Convention on the Rights of the Child establishes a description of particularities of the evolution of the matter in that country, prior and after the ratification of this landmark international treaty. The Spanish experience runs parallel to the European one, with the $19^{\text {th }}$ century as a crucial period in the variation of the consideration of children and teenagers. Indifference gives way to worry and this change is reflected in legal acts centered in the protection of those collectives and there is also a precedent of the consideration of children as persons capable of making decisions in matters of their own interest, in the beginning of the Second Spanish Republic. The 1936-1939 Civil War and the dictatorship of general Franco-Bahamonde suppose a hiatus for democracy and freedom in the country and only with the 1978 Spanish Constitution Spain will return to the democratic path. The beginning of the new Spanish democracy runs parallel with the preparation of the 1989 Convention and there are many common points in the list of general principles and the objectives. Both legal texts introduce ideas as the equality of every children and the child's best interest and will have a mandatory influence in the transformation of the Spanish Family and Childhood Law. However, efficacy of legal reforms should be always periodically analyzed to determine if their directive principles have been truly applied or not and, if not, investigate and search for the reasons, systemic or conjunctural of the failure. 